\title{
Erratum
}

Chromosoma (1996) 105:190-196

\section{Genomic dispersion of 28S rDNA during karyotypic evolution in the ant genus Myrmecia (Formicidae)}

Hirohisa Hirai $^{1}$, Masa-Toshi Yamamoto ${ }^{2}$, Robert W. Taylor ${ }^{3}$, Hirotami T. Imai ${ }^{4}$

${ }^{1}$ Primate Research Institute, Kyoto University, Inuyama, Aichi-ken 484, Japan

2 Department of Applied Biology, Kyoto Institute of Technology, Matsugasaki, Kyoto 606, Japan

${ }^{3}$ Division of Entomology, CSIRO, Canberra, ACT 2601, Australia

4 National Institute of Genetics, Mishima, Shizoka-ken 411, Japan

Received: 22 March 1996; in revised form: 3 June 1996 / Accepted: 4 June 1996

Figure 2a-r of the above article is reprinted here at the request of the author because the print quality was not up to standard in the issue. 


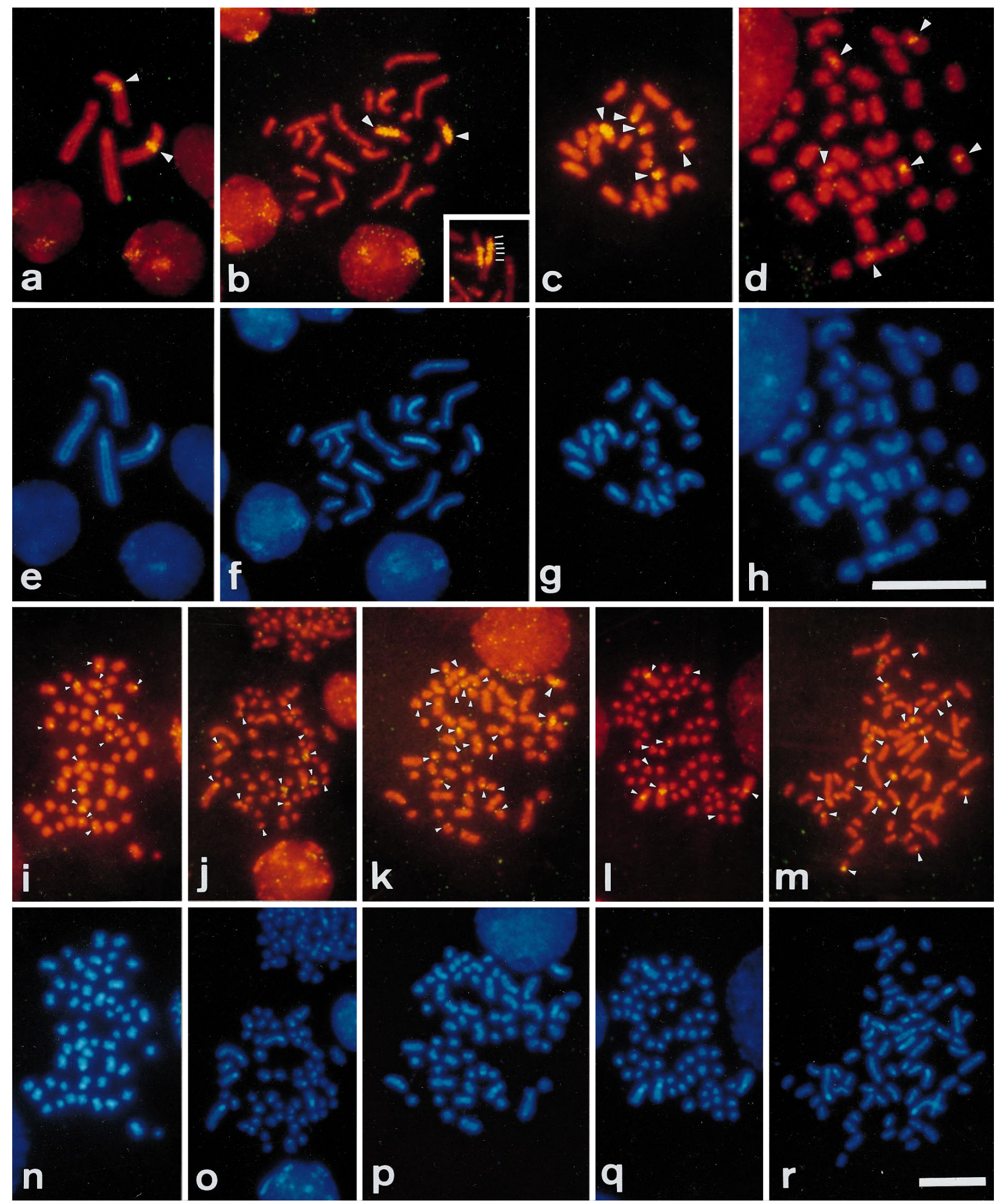

Fig. 2a-r. Localization of 28S rDNA (white arrowheads) by fluorescence in situ hybridization of preparations from various $M y$ rmecia species with different chromosome numbers. a-d, i-m Hybridized in situ and propidium iodide-stained chromosomes. e-h, n-r 4',6-diamidino-2-phenylindole-stained chromosomes. a, e $M$. croslandi $(2 n=4)$. b, $\mathbf{f}$ M. pilosula $(2 n=22)$; the inset in b

shows repeated layers of rDNA-positive signals in C-band. $\mathbf{c}, \mathbf{g} M$. pilosula $(2 n=23)$. d, h M. gulosa $(2 n=38)$. i, n M. forficata $(2 n=52)$. j, o $M$. mandibularis $(2 n=56)$. k, p Myrmecia sp. cf. $M$. arnoldi $(2 n=60) \mathbf{i}, \mathbf{q} M$. occidentalis $(2 n=64) \mathbf{m}, \mathbf{r}$ M. simillima $(2 n=70)$. Bars represent $10 \mu \mathrm{m}$. The long bar in $\mathbf{h}$ applies also to $\mathbf{d}$; the short bar in $\mathbf{r}$ to all other panels 